\title{
ENVIRONMENTAL CHALLENGES, TECHNICAL SOLUTIONS AND STANDARD OPERATING PROCEDURES FOR DATA COLLECTION IN PHOTOGRAMMETRIC STUDIES TOWARD A UNIFIED DATABASE OF OBJECTS AND FEATURES IN UNDERWATER CAVES IN MEXICO
}

\author{
Fortin, Julien ${ }^{1}$; Meacham, Sam ${ }^{1}$; Rissolo, Dominique ${ }^{2}$; Le Maillot, Christophe ${ }^{1}$; Devos, Fred ${ }^{1}$ \\ ${ }^{1}$ Centro Investigador del Sistema Acuífero de Q Roo (CINDAQ A.C.), Puerto Aventuras, Q Roo, Mexico; info@cindaq.org \\ ${ }^{2}$ Qualcomm Institute, University of California, San Diego, La Jolla, CA, 92093-0436, USA
}

KEY WORDS: Photogrammetry, underwater, caves, field methods, Mexico.

\begin{abstract}
:
Mexico's Yucatan Peninsula is predominantly formed as a karstic terrain on limestone substrates where hundreds of kilometres of now submerged caves constitute extended networks of galleries partially accessible through cenotes, or karstic windows. These cenotes and underwater caves contain numerous objects of great biological, historical, cultural and/or paleontological significance: human and animal remains, ancient Maya artifacts, ancient mines and traces of early human activities. Photogrammetric studies by technical divers allow researchers to access a vast array of data pertaining to objects or features which would otherwise remain inaccessible. However, the harshness of the study environment poses technical acquisition challenges which must be specifically addressed. This paper introduces these environmental challenges, including the geological properties of local caves, bottom composition, ceiling percolation and topography, as well as the intrinsic difficulties linked to the absence of light and the limited time available in an underwater work setting. Technical acquisition solutions, setups and standard operating procedures are then proposed to overcome these challenges. A further consideration is added pertaining to the overall goal of creating a global database for the study objects, thus requiring a unified format for all data: unique identifier, geolocation, minimum resolution, and the like. The authors, focusing on the fieldwork and the data-collection aspect of the photogrammetric study, rather than the data processing and metrological steps, define a new image acquisition methodology built around a compact and replicable setup which will allow the documenting of objects previously out of reach of traditional setups.
\end{abstract}

\section{INTRODUCTION}

\subsection{Environment}

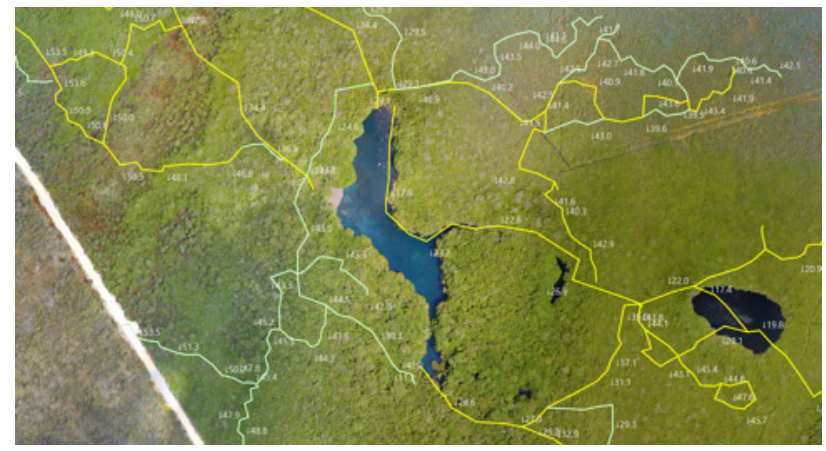

Figure 1. (A) An extensive underwater cave network connected to a karstic window in the Yucatan Peninsula (CINDAQ)

The Yucatan Peninsula is predominantly formed as a karstic terrain on limestone substrate. The majority of these strata, laying on igneous and metamorphic basement rocks, originated in the Cretaceous period, where carbonate sediments from coral reefs and seashells were deposited on the ocean floor (VázquezDomínguez, 2010). Acidity generated at the surface in later periods featuring lower sea levels, notably rain percolating through the more superficial soil layers, lead to the formation of dry underground caves (Smart et al., 2006) sporting numerous speleothems. Besides this chemical formation process, the underground landscape has been shaped through the emergence of mechanical fracture zones (Bauer-Gottwein, 2011), among them the one resulting from the shock wave around the Chicxulub impact crater, the landing site of the meteorite determined to be the cause of the great Cretaceous-Tertiary extinction (Alvarez, 1980). Along these fault lines, or at locations where the ceilings collapsed due to external factors, karstic windows, locally called cenotes, have formed and give access to the result of all these combined forces: one of the most extended cave systems in the world (see figure 1), now mainly flooded since the sea level rises following the end of the Pleistocene (Smart et al., 2006).

\subsection{Objects and features}

The caves of the Yucatan Peninsula have been, at times of lower sea levels, used as shelters and access points to ground water by animals and humans alike. Furthermore, traces of sustained human activities, for instance ochre mining, have been documented in the now submerged caves of Quintana Roo (McDonald et al., 2020). These objects of great biological, historical, cultural and paleontological significance (figure 2) need to be documented, not only to provide new data to scientists unable to access them due to their location, but also to preserve as much information as possible before eventual destruction through time, cave collapses or human-induced degradation.
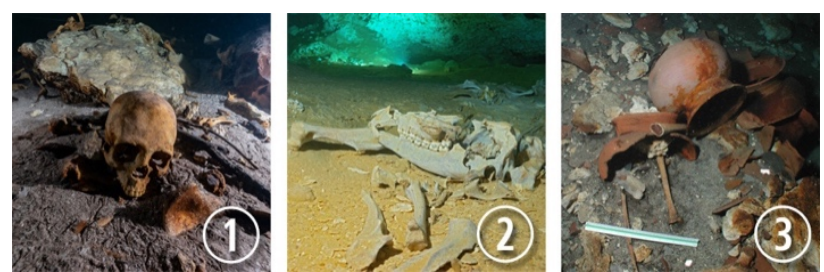

Figure 2. Objects and features to be documented include (1) Human and (2) animal remains, (3) artifacts (CINDAQ) 


\subsection{Geo-located database}

While studying a single feature in an underwater cave through photogrammetry can yield valuable data for scientific research and contributes to the understanding of greater topics, multiplying the number of studies and connecting them through a geo-located database can considerably increase the scientific potential.

Not only does this create more data points, but the integration of these data points into a Geographic Information System (GIS) unlocks new possibilities for discovering correlations between the objects and their surroundings, thus adding contextual information which would likely be either absent or undetectable through a single photogrammetric study.

Tying in all collected data in such a GIS database requires the definition and implementation of strict Standard Operating Procedures and unified formatting to be able to compare and link the data points: unique identifier (UID), precise geolocation through GPS coordinates at the surface and line survey underwater, minimum resolution and standard lighting for the photogrammetric data collection, and the like.

Furthermore, the overall goal of gathering as much data as possible to feed a GIS-based database requires, in order to yield useful results in a realistic time frame, a collection setup and methodology which can be deployed inexpensively, fast and easily without falling under a minimal quality threshold.

\section{ENVIRONMENTAL CHALLENGES}

\subsection{Absence of natural light}

The first and most obvious characteristic of the underwater cave environment as it relates to image collection is the absence of natural light. While sunlight can still be found in what is frequently referred to as the "cavern zone" of the underground systems, close to the exits, the actual cave beyond the daylight zone constitutes a working environment of complete obscurity.

Image acquisition therefore requires sufficient artificial lights to be brought to the photogrammetric study site. As opposed to a setting on land, installing fixed light in a cave can be logistically challenging or impossible, be it for lack of stable surfaces or to avoid the risk of stirring up sediments (section 2.2). Ideally, light intensity also needs to be adjustable to adapt to different reflectance characteristics of the floor, wall and ceilings of the cave. An intense light might work well for a dark-coloured cave but create an over-exposure in a white passage. Finally, the total absence of natural light and complete dependence on artificial sources potentially creates difficulties in shadow management a single light possibly generating darker areas in the acquired images, which in turn can impede the model generation when processing the data.

\subsection{Sediments}

The geological nature of the Yucatan Peninsula's underwater caves, with their main conduit mainly formed by the dissolution of limestone substrates, induces the presence of dissolution products in the form of various sediments. Additionally, their hydrological nature leads, in some cave segments, to an accumulation of particles and materials carried by the underground rivers both from openings and upstream sections, similarly to an alluvion phenomenon. Finally, in cave areas where the water level, at current or past times, is or was lower than the ceiling height, formation of calcite rafts at the water surface and the subsequent collapse of this thin layer under its own weight or external disturbance has generated an accretion of calcite flakes on the cave floor. These various sediments create a new type of challenge for photogrammetric studies in underwater caves, which requires exact assessment depending on the nature and location of the sediments, as well as the definition of a technical solution tackling this challenge.

2.2.1 Different types of sediments: The main sedimentrelated property carrying critical relevance for photogrammetric studies is the grain or particle size. Broadly speaking, smaller particles, being lighter, tend to be easily disturbed by natural (flow, partial collapses, rainwater percolation through the soil) and human (fin kicks, lack of buoyancy control, impact of tools needed for the studies) factors. The ISO 14688-1:2002 norm defines the accepted nomenclature for sediments according to their grain size - Table 1 lists the sediments with a grain size smaller than $0.063 \mathrm{~mm}$ in diameter, as these are the soil types most likely to present technical challenges during the underwater photogrammetry process.

\begin{tabular}{|l|l|l|l|l|}
\hline \multicolumn{2}{|l|}{ Name } & \multicolumn{2}{|c|}{ Size range $(\mathrm{mm})$} \\
\hline Coarse soil & Sand & Fine & FSa & $0.063-0.2$ \\
\hline Fine soil & \multirow{2}{*}{ Silt } & Coarse & CSi & $0.02-0.063$ \\
\cline { 3 - 5 } & & Medium & MSi & $0.0063-0.02$ \\
\cline { 3 - 5 } & & Fine & FSi & $0.002-0.0063$ \\
\cline { 2 - 5 } & Clay & Cl & $\leq 0.002$ \\
\hline
\end{tabular}

Table 1. ISO 14688-1:2002 soil classification for types of underwater cave sediments susceptible to interfere with photogrammetric studies

Besides the limited amount of energy and impact required to stir them up, these fine particles will, once disturbed, also require a larger time interval to settle down. Depending on the project, the duration needed for sediments to decant will either reduce the amount of time available to gather data during the current dive, or even, in the case of clay-like particles with a diameter inferior to $0.002 \mathrm{~mm}$, warrant aborting the dive. Figure 3 (Jablonski, 2001) illustrates the designated settling time in stagnant water of various sediment types: up to five hours can be necessary, after stirring up fine clay, to recover enough visibility for the photogrammetric process to occur.

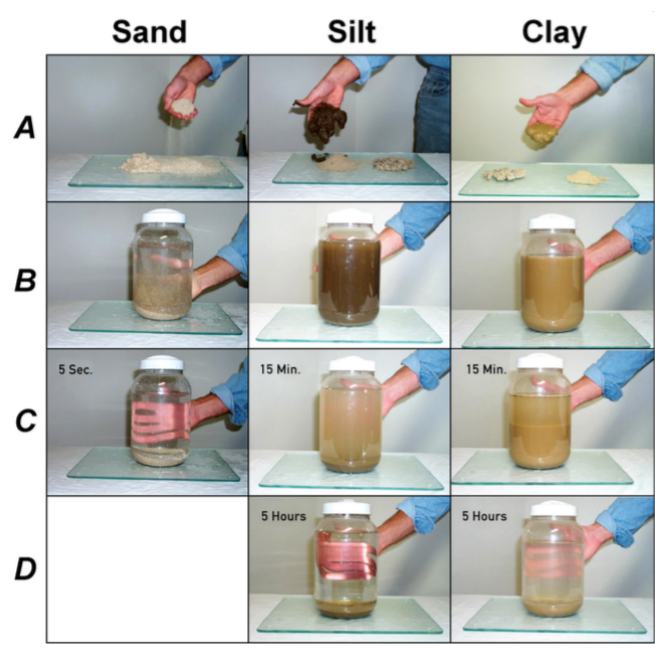

Figure 3. (A) Sand, silt, and clay as dry sediments. (B) Sand, silt, and clay mixed in a column of clear fresh water. (C and D) Mixture of sand, silt, and clay after the designated settling time in stagnant water (Jablonski, 2001) 
2.2.2 Different types of ceilings and percolation: While we intuitively think of sediment-linked problematics in underwater caves to be caused by floor particles being stirred up, a second phenomenon can also drastically decrease visibility: ceiling percolation. Fine particles either generated by the original cave dissolution or the hydrological movements over large period of time can be trapped in the ceiling's asperities and start falling at the slightest mechanical disturbance. Additionally, the carbon dioxide contained in exhaled gas of divers, especially using opencircuit apparatus, can trigger a new dissolution of the limestone in the ceiling, likewise resulting in fine particles falling and rendering the study site impossible to model for an extended period of time. Finally, it is to be noted that even a small amount of percolation, while not impairing visibility around the object, will very quickly produce, if not handled properly, substantial backscatter, making all or parts of the images unusable for building the model, as can be seen in figure 4 .

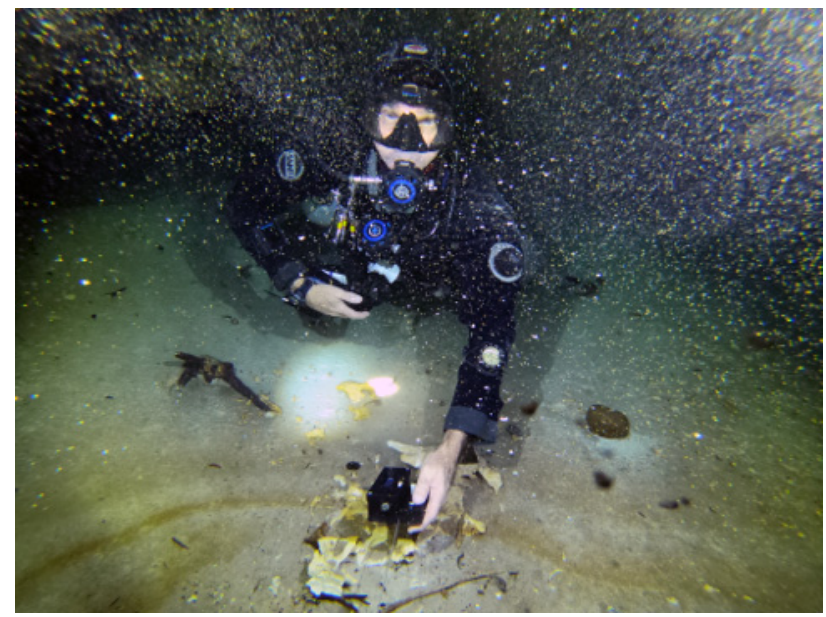

Figure 4. Percolation in an underwater cave: sediments dislodged from the ceiling and creating intense backscatter (CINDAQ)

2.2.3 The importance of flow: Understanding submerged cave systems as underground rivers moving large expanses of water is critical to the execution of photogrammetric studies. Scientific divers need to consider the double nature of sediment movements in the cave: not only vertically, through gravity, but also horizontally through flow. Any disturbance upstream of a study object will result in potentially large amounts of sediments traveling with the water flow to the location of the work to be performed. Incorporating flow directions into the photogrammetric site organization is therefore of paramount importance and will be addressed in the definition of the Standard Operating Procedures in section 3.

\subsection{Topography: restrictions, cracks and low ceilings}

An underwater cave being, by definition, an overhead environment, the height of the ceiling is one of the critical topographical features that can increase the difficulty of photogrammetric studies. A low ceiling of one meter or below creates at least two challenges, one pertaining to the tool being used, and the second one to the work itself. A limited space between the object and the ceiling implies the need for a compact setup that can not only fit in the available gap, but also be comfortably manoeuvred to cover all necessary angles. Furthermore, low ceilings increase the probability of impacting the cave by restricting the scientific divers' movements, thus resulting in the array of sediment-linked problems highlighted in previous sections.
A second challenge is frequently posed by the location of the study objects on the cave floor, which is rarely, if ever, a perfect, flat and solid surface. Bones are regularly found in depressions or cracks, which might in some case have prevented dispersion through hydrological flows, but also possibly limits the angles available for image gathering. A typical, bulky photogrammetry setup often prevents data collection in such settings.

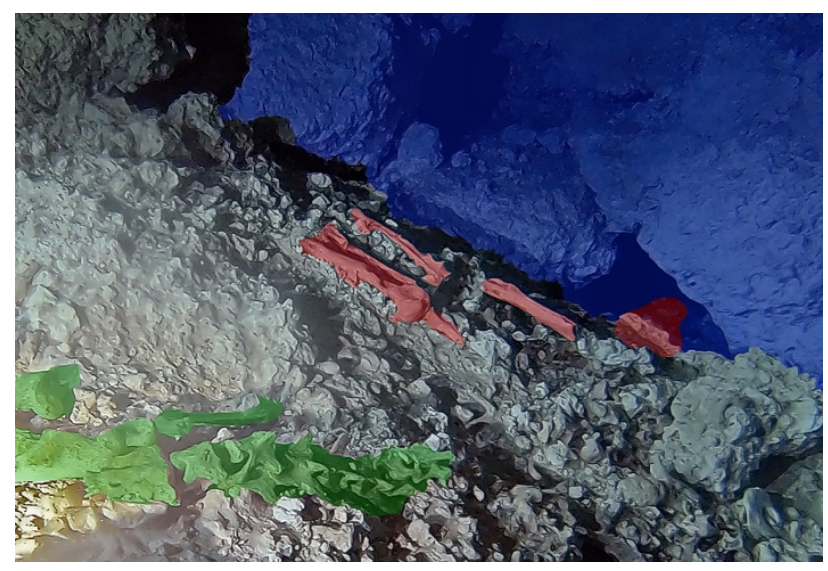

Figure 5. While some bones are easily accessible for photogrammetry (highlighted in green), others (highlighted in red) are located under an overhanging rock (highlighted in blue), and cannot be documented using standard photogrammetric setups (CINDAQ)

While the objects and features of photogrammetric studies in an underwater cave might or might not be in a large space with easy access to different view angles, an important point to be noted is the fact that these objects' location needs to be reached by the scientific divers to collect data. Even in the ideal case of, for instance, bones being found in a large room with solid floor and a high ceiling, the passages leading to them might, and often will, present minor or major restrictions through which the team and photogrammetric equipment needs to travel safely - further highlighting the need for a compact setup.

\subsection{Time constraints}

2.4.1 Availability of breathing gas: When working in a flooded tunnel without direct access to the surface, frequently hours away from the nearest exit, and carrying a limited amount of breathing gas, the time available for data gathering during the photogrammetry process understandably constitutes a critical factor - giving the term "deadline" a very real meaning. Breathing gas will be obviously required not only for the actual data collection, but also for traveling to and from the site, for setting up the collection area if needed, and for a substantial safety reserve set aside for possible contingencies. It is therefore of paramount importance to minimize the time needed to reach the study object by making the equipment as portable as possible, but also to define clear Standard Operating Procedures avoiding preparation or non-value adding tasks in the cave to maximize the amount of gas, and thus time, dedicated to the actual photogrammetric data collection - while maintaining a conservative safety reserve. 
2.4.2 Impact of collection time on the cave and the study object: Beyond considerations of gas availability, the percolation phenomenon described in section 2.2.2, while it can be mitigated with proper procedures, equipment choices and skills, as will be described in section 3, is unavoidable in fine for most caves. The more time is spent around a study object or feature, the more percolation is likely to be generated - and reducing the data collection process duration becomes an important factor for image quality improvement. Last, but not least, despite the fact that image collection is ideally a non-invasive process, the probability of impacting the object through accidental contact or disturbance of the geological features increases drastically with the amount of time spent by divers in its surrounding.

\subsection{Logistics and redundancy}

The sum of all environmental challenges within the underwater caves, coupled with the difficulty of access of the caves themselves, often imply a high level of logistical complexity until the photogrammetry team reaches the study site in conditions conducive to the work to be executed. Cancellation, at that point, of the work due to equipment failure represents a substantial waste of resources and must be prevented by aiming for the maximum possible level of redundancy. The technical setup to be defined needs to allow for optimal modularity around components for which backup can conveniently be carried.

\section{TECHNICAL ACQUISITION SOP}

Given the nature of anastomosing morphologies and the fragility of deposits and features, ROV or AUV platforms for photogrammetric documentation are often not practical in caves. Tethered systems can be unmanageable and destructive, and thrusters can disturb cave sediments (particularly in narrow spaces). Even small AUVs with sophisticated SLAM autonomy, collision avoidance capability, and variable buoyancy control would be challenged by these extremely complex environments - some requiring divers to remove their tanks to fit through restrictions. Therefore, survey and digital documentation conducted by trained cave divers remains the most effective approach.

Numerous studies focus on the unique challenges of SfM photogrammetry underwater, as well as the refinement of techniques to produce geometrically accurate models of submerged archaeological deposits or objects (e.g. Drapp 2012). Photogrammetric documentation in submerged caves, to date, has not kept pace with the widespread adoption (and adaption) of techniques by archaeologists working on open-water sites. Notable subterranean examples include a stereo vision approach to cave mapping (Weidner 2017) and the novel above- and below-water photogrammetric survey of a semi-submerged cave (Nocerino et al. 2019; see also Nocerino and Menna 2020). García Sedano (2014) vectorized photomosaics to enable spatial analyses of submerged Maya deposits in a cenote in Yucatan. In terms of SfM photogrammetry in region, the Hoyo Negro Project represents a large-scale, multi-year effort to enable virtual access to submerged Late Pleistocene-Early Holocene deposits via a multi-resolution digital twin of the cave (Rissolo et al. 2018). The systematic survey and photogrammetric documentation of La Mina (see MacDonald et al. 2020) represents a coordinated effort to expand the use of image-derived data for a range of analyses involving the region's submerged cave systems.

In certain cave contexts and deployment scenarios, the use of a full-frame sensor camera and appropriate lens and port combinations is preferred. Equipment selection is often a balance between achieving the highest resolution (and corresponding highest point density) possible, while not encumbering the cave diver with the camera and lighting setup itself. Camera, lens, and port (dome or flat) combinations for object-, deposit-, or tunnelscale imagining have been informed by experimentation and available comparative studies (e.g. Menna et al. 2016; Menna et al. 2017). While a critical discussion on issues related to optical aberrations, field of view, depth of field, and the like are beyond the scope of this study, they are nevertheless considered in the selection of task-specific equipment. The use of the GoPro HERO8 (described below) accepts certain limitations (in terms of optics and sensor), though it should be noted that current iterations benefit from a flat port (see Menna 2017:482 for explanation).

The superior performance of full-frame sensor cameras (mirrorless or DSLR) with professional quality lenses notwithstanding, a compact and manoeuvrable camera and lighting setup is better suited to certain submerged caves sites (given the environmental conditions and logistical parameters already discussed). It is then incumbent upon the scientific divers to develop and deploy a compact setup that produces the best possible results.

\subsection{Compact setup}

Environmental challenges highlighted in the previous sections underline the urgent need for a more compact setup for underwater cave photogrammetry studies. Reducing the footprint of the system used has been the focus of the authors' efforts and resulted in a completely new configuration based around a standard GoPro HERO8 camera, as described in figure 6.

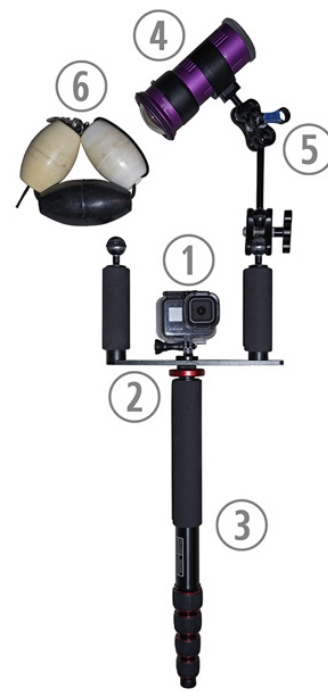

Figure 6. Compact photogrammetric setup for underwater caves including: (1) GoPro HERO8 camera ; (2) Aluminium tray ; (3) Telescopic, aluminium mono-tray with purge holes ; (4) 15,000

Lumen Keldan light ; (5) Adjustable light arm with two ball bearings ; (6) Floats with bolt snap to ensure neutral buoyancy (CINDAQ)

The resulting system has been extensively tested and refined in Mexican caves to generate around twenty different models, to arrive at the configuration presented above. The reduced footprint is best illustrated by comparing, side-by-side, the new, compact, underwater-cave specific GoPro photogrammetry setup to the high-performance, large-volume DLSR photogrammetry setup used in the authors' previous work. Figure 7 demonstrates the substantial volume reduction and manoeuvrability increase. 

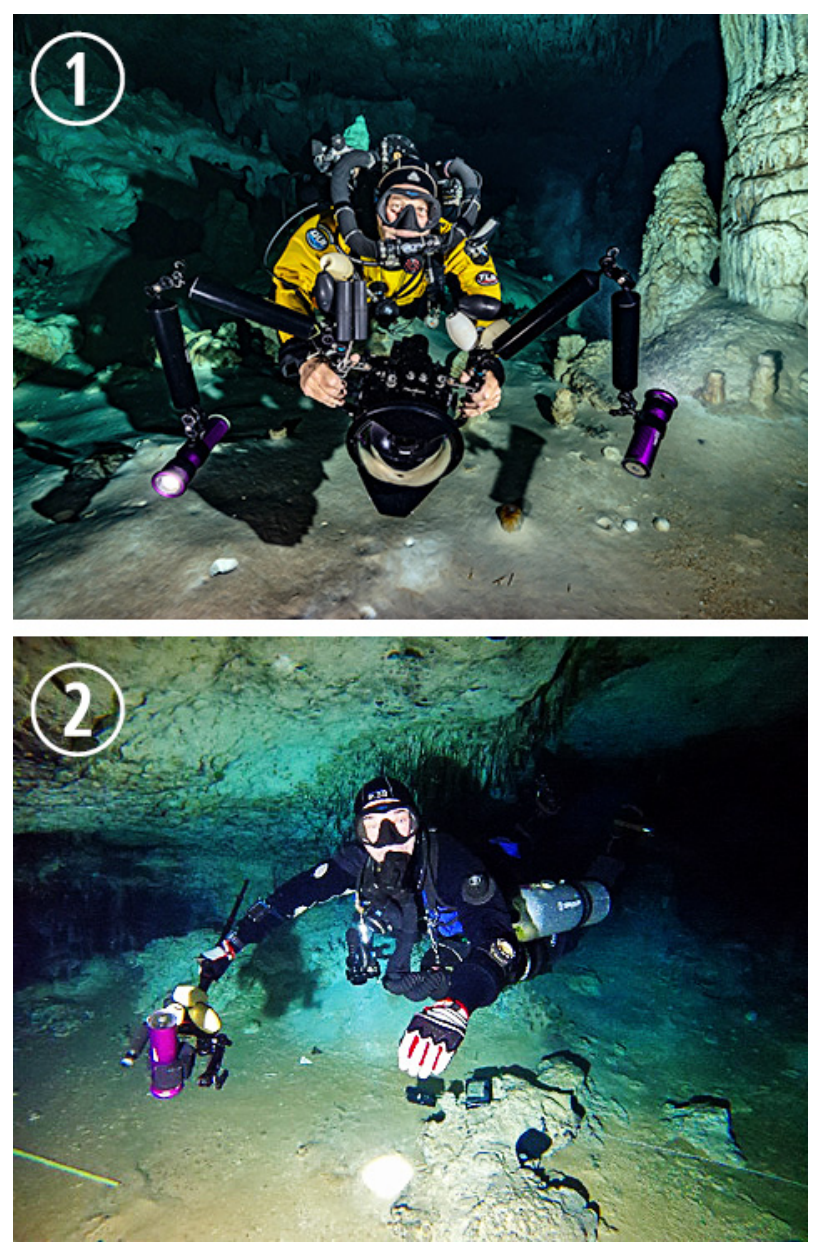

Figure 7. Comparative pictures of: (1) high-performance, largevolume DLSR photogrammetry setup ; (2) compact, underwater-cave specific GoPro photogrammetry setup (CINDAQ)

3.1.1 Camera: A GoPro HERO8 camera was used for our tests. The camera measures $62.3 \mathrm{~W}$ x $44.9 \mathrm{H}$ x 33D $(\mathrm{mm})$. Photo time lapse mode was used for image capture with the following settings in place (Table 2).

\begin{tabular}{|l|l|l|}
\hline General settings & Lens & Narrow (27mm) \\
\cline { 2 - 3 } & Interval & 1 Second \\
\cline { 2 - 3 } & Format & Photo \\
\cline { 2 - 3 } & Output & Standard (JPEG) \\
\cline { 2 - 3 } & Zoom & $1 \mathrm{x}$ (default) \\
\hline Protune Settings & EV Comp. & 0 \\
\cline { 2 - 3 } & White balance & $5500 \mathrm{~K}$ \\
\cline { 2 - 3 } & ISO min. / max. & $100 / 400$ \\
\cline { 2 - 3 } & Sharpness & High \\
\cline { 2 - 3 } & Color & Flat \\
\hline
\end{tabular}

Table 2. Technical settings of the GoPro HERO 8 camera used for the compact underwater cave photogrammetry setup

Standard output (JPEG) was used since RAW output increases the capture interval to 5 seconds from 1 second. The one second interval allows for more fluid movement of the camera over a site and reduces arm fatigue when the camera is held at its furthest extension. Mini SD cards with a 32-gigabyte capacity provided more than enough storage capability for the number of photos captured. Due to its compact size, we were able to carry a backup GoPro on every dive as redundancy in case of flooding, or battery discharge. The diver not having the task of photogrammetry was able to use the backup camera to document the general area of the site.

3.1.2 Lighting: A single Keldan Video 4X $10000 \mathrm{~lm}$ CRI 85 light was used to illuminate the sites that were documented. The specifications for the light are:

\begin{tabular}{|l|l|}
\hline Luminous Flux max. / min. & $10,000 \mathrm{~lm} / 200 \mathrm{~lm}$ \\
\hline Candlepower max. / min. & $4300 \mathrm{~cd} / 90 \mathrm{~cd}$ \\
\hline Burn time max. / min. & $35 \mathrm{~min} / 850 \mathrm{~min}$ \\
\hline Beam angle & $110^{\circ}$ (in water) \\
\hline Correlated Color Temp. & $5,600 \mathrm{~K}$ \\
\hline Diameter $(\mathrm{Head}) /$ Length & $7 \mathrm{~cm} / 17 \mathrm{~cm}$ \\
\hline Weight (Dry/Wet) & $0.63 \mathrm{~kg} / 0.20 \mathrm{~kg}$ \\
\hline Max depth rating & $200 \mathrm{~m}$ \\
\hline
\end{tabular}

Table 3. Technical specs of the Keldan Video 4X $10000 \mathrm{~lm}$ CRI 85 light used for the compact underwater cave photogrammetry setup

The light provided ample coverage for the $27 \mathrm{~mm}$ focal length of the camera, and enough burn time to cover more than one site per dive. The settings for the light were put on the lowest of the range of intensity and were varied depending on the colour of the bottom sediment. Like the GoPro, the compact size of the Keldan light allowed us to have a backup on each dive in case of failure in the primary unit.

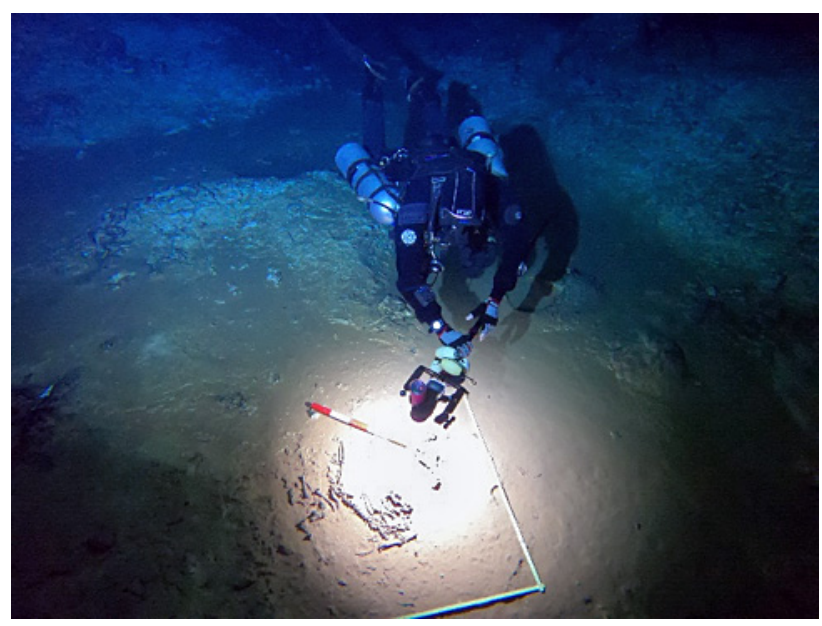

Figure 8. The Keldan lighting during a photogrammetric data collection process in an underwater cave (CINDAQ)

3.1.3 Monopod and dual handle tray: Using a monopod as a camera support can help overcome the challenge of low ceilings described in section 2.3. Such a monopod, provided it features sufficient length, creates distance between the camera and the diver, thus mitigating the silt-out and percolation issues. A telescopic, aluminum monopod, allowing for a more compact configuration during transport, has been identified by the authors to be the ideal camera support for their application. It is worth noting that each of the tubes building the telescopic monopod needs to be drilled before in-water usage: without this preparation, surrounding water pressure will compress the gas trapped in the tube, preventing full deployment.

Though monopods and dual handle trays are not commonly combined on land, underwater cave photogrammetry can benefit from their cumulative advantages: the pros mentioned here for the monopod being perfectly complemented by the ability of the 
tray to host foldable arms which can be used to accommodate a video light and adjust its orientation precisely. As an added benefit, the tray provides several attachment points to which floats can be connected to ensure neutral buoyancy, thus reducing torque and fatigue, and increasing accuracy when the monopod is fully deployed. In order to fit the GoPro HERO8 camera to both the monopod and the tray at the same time, a solution had to be found to compensate for the limited depth of the threaded hole in the base of the standard GoPro casing, as well as the limited length of the final bolt of the monopod. A conversion thumb screw, or 1/4 inch adapter rail extension, was added between the monopod and the tray, thus increasing the bolt length to be go through the tray material and screw into the GoPro casing base, as shown on figure 9 .

\subsection{Time optimization}

Having established earlier that time constitutes a critical parameter for the photogrammetric process in an underwater cave environment, notably due to the limited amount of breathing gas available, but also to percolation and potential impact to the cave and study object, Standard Operating Procedures need to put time optimization at the center of the collection team's priorities. Nonessential tasks have to be avoided as much as possible to make the most of the data collection time available.

3.2.1 Pre-dive preparation: Proper planning and preparation are essential to ensure that the time spent in the water can be dedicated to the data collection process. One of the advantages of the compact setup described in section 3.1 is that it can be transported as is, inside a bag clipped to the diver's equipment once the telescopic monopod has been retracted. Trials in the water by the authors have shown that transporting the monopod, the camera and the tray separately, i.e. following a Completely Knocked Down (CKD) approach, substantially increased the risk of losing parts as well as the preparation time after reaching the photogrammetric study site, while not presenting worthy transportation advantages due do the compactness of the designed setup. The light connected to the tray can also be transported as is, provided the support arms are folded to reduce the overall size. Preparing the magnetic supports with the Unique Identifier (UID), the date and the north arrow (see section 3.4.2) in the lab and transporting them in separate flexible containers also speeds up in-water preparation.

Finally, determining the different zones for the photogrammetric work (see section 3.3) using maps and surveys prior to the dive streamlines team communication and reduces time pressure. It also and importantly allows efficient role assignment to the individual divers participating into the data acquisition effort.

\subsection{Zoning}

Clearly organizing the photogrammetric study area into different zones has proven to be of crucial importance to avoid unnecessary silting out of the object's surrounding, both through disturbance of floor sediment and through percolation, for reasons mentioned in sections 2.2.1 and 2.2.2. Adequate zoning should take flow direction into consideration, and circumscribe non-data-collecting tasks to locations situated downstream of the study area. Separating the different steps of the overall process into different spaces, more importantly, is a valuable way of limiting the risk of impacting the objects of features of interest.

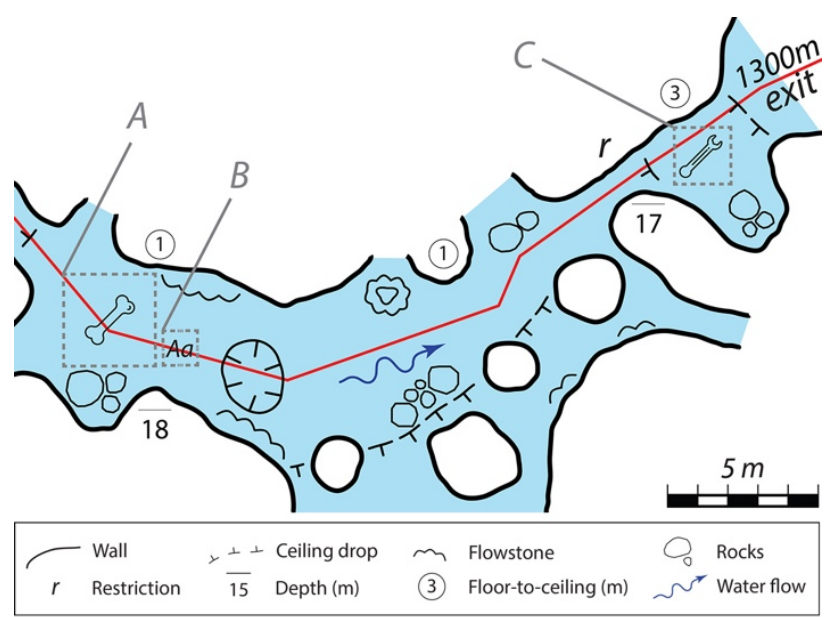

Figure 10. Cave map illustrating the different photogrammetric study areas: (A) the acquisition zone (downstream, $1 \mathrm{~m}$ floor-toceiling height, $18 \mathrm{~m}$ depth) ; (B) the legend zone (next to the photogrammetry zone, downstream); (C) the preparation zone (further downstream, $3 \mathrm{~m}$ floor-to-ceiling height, before the restriction " $r$ ") (CINDAQ)

3.3.1 The acquisition zone: The immediate vicinity of the object should only be approached when collecting the images, and not for peripheral tasks like setting up the equipment. Within the scientific dive team, the one in charge of data acquisition and coming near or entering the acquisition zone should be able, besides maintaining the situational awareness necessary for team safety, to focus on the process and the item. This increases image quality and avoids disturbing particles, but also ensures the least possible impact on the objects being studied. A scientific diver setting up the camera equipment too close to the subject could, for instance and while their attention is diverted to their task, contact and damage fragile bones with their fins. An efficient method to delimit the acquisition zone is the use of yellow folding rulers to create either a L-shaped half frame or a complete rectangular frame around the acquisition zone, thus serving the dual purpose of incorporating a scale into the images and clearly delimiting the main work area, as is shown in figure 11.

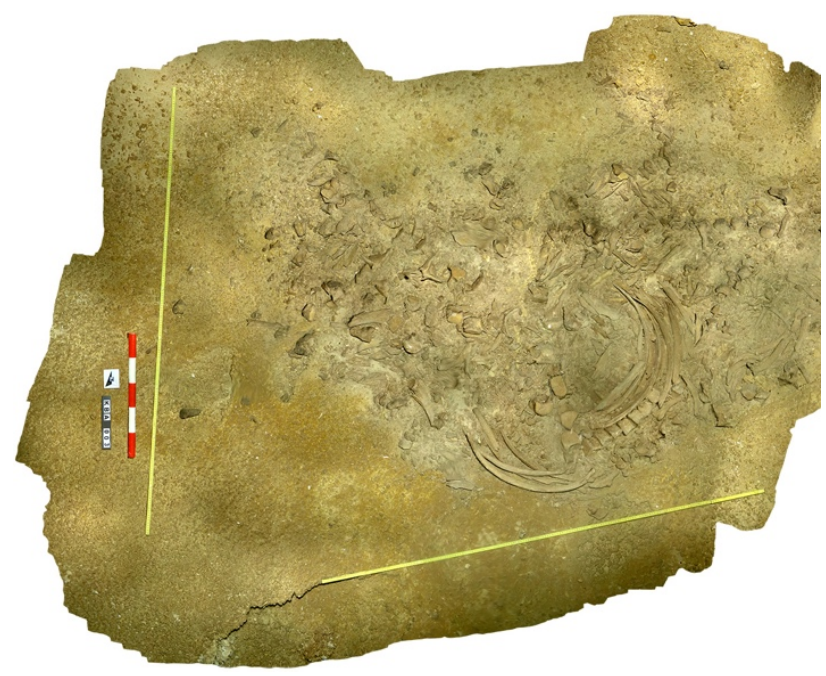

Figure 11. Example of an acquisition zone, including two yellow folding rulers for scale and delimiting the work area. N.B.: the legend zone can be seen on the left (CINDAQ) 
3.3.2 The legend zone: Section 1.3 established the need for a unified data format to create data points that can be linked through a GIS database. Precise geo-location of the current study object or feature requires including a known survey point into either the photogrammetry or the legend zone. The legend zone is the area containing the metadata of the study object, including but not limited to : a North arrow (for orienting the subject not only in relation to its surrounding, but also to the global system in which it is fine), the date (to be able to establish a baseline and assess the evolution of the subject over time if necessary), a unique identifier (UID), a scale (ideally in the form of a colored yard stick to serve the dual purpose of metric scale and color balance when post-processing). The authors have found magnetic bases and magnetized alphanumerical tiles to constitute an ideal solution for this purpose. If possible, given the topography, the legend zone should be situated in the immediate vicinity of the acquisition zone, directly outside the frame described in section 3.3.1, on its downstream side.

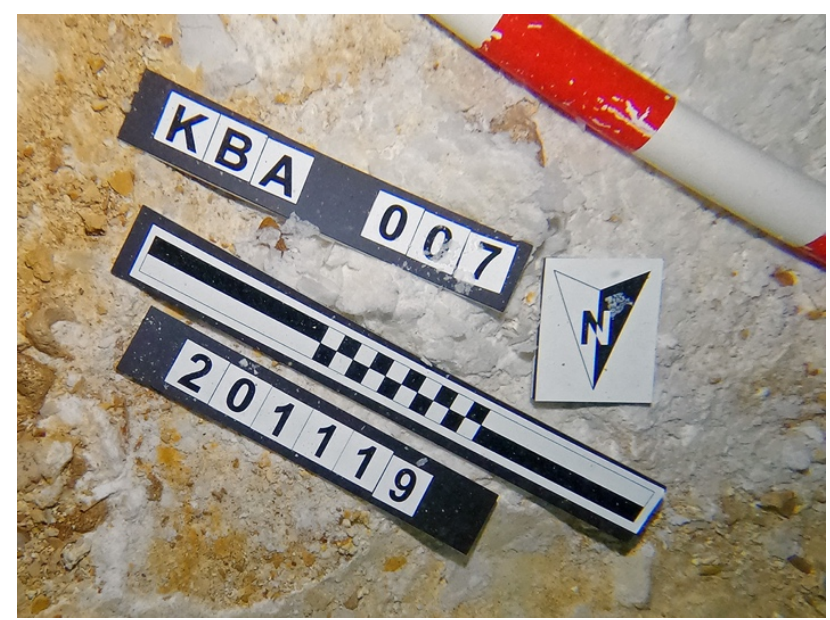

Figure 12. Example of a legend zone including magnetic bases, magnetized alphanumerical tiles with the Unique Identifier (UID), a North arrow, the date, and two scales (CINDAQ)

3.3.3 The preparation zone: Extensive travel of up to several hours through flooded tunnels is routinely necessary to access the study object. The photogrammetry setup described in section 3.1 , as well as the metadata markers described in section 3.3.2, need to be transported in a folded state or in separate flexible containers. A specific zone, well outside the acquisition and legend areas, and necessarily downstream from both, is defined for final deployment of the setup before approaching the object. This preparation zone can also be used to store dive equipment required to reach the area, like additional gas cylinders, Diver Propulsion Vehicles (DPVs), or transportation bags for the photogrammetric tools. In complex cases, several preparation zones can be required: one to store the DPVs and additional breathing gas cylinders, and one for the deployment of the setup.

\subsection{Dive equipment choice strategies}

Attention should be paid by the scientific divers to their dive equipment choice strategies.

Open-circuit, self-contained underwater breathing apparatus (SCUBA) is widely used for all kinds of underwater scientific work and can be perfectly adequate tools depending on circumstances and topography. While it is quite simple and reliable in nature, the large amounts of bubbles released into the surrounding water with every exhalation can make it more prone to triggering heavy percolation in fragile cave environments.
Closed-circuit rebreathers (CCR) solve the issue by recycling exhaled gas through a loop and counter-lung, drastically reducing both bubbles and percolation. They also potentially reduce the amount of gas needed, or increase the time available, thus mitigating the time constraints mentioned in section 2.4. The vital necessity to closely monitor oxygen partial pressure, however, can potentially be an issue when the concentration of the diver is to be focused on the photogrammetric data collection process.

The use of Passive, Semi-Closed Rebreathers (PSCR) has been found by the authors to constitute a possible solution combining limited percolation with an ease-of-use letting the divers concentrate on photogrammetry. These systems being keyed in to the divers' respiration, and not including on-board oxygen which could result in hyperoxia in case of a malfunction, equipment awareness can be maintained without impeding the concentration necessary for the scientific study, while reducing time constraints and disturbance of sediments.

Regardless of the elected strategy, ample breathing gas reserves need to be planned and either carried by the team or pre-installed to provide safety to all team members.

\subsection{Teamwork and personal skills}

As a last note, and merely touching upon an aspect reaching beyond the scope of the current article, the authors would like to underline that photogrammetric data collection in an underwater cave is a task that can only be built safely upon solid personal dive skills and team procedures.

Buoyancy control and precise kicking techniques are essential to preserve both the cave and the study objects. Situational awareness of environment, equipment and team is a sine-qua-non condition for ensuring divers safety while a part of their attention is diverted to the scientific work. Well-defined diving protocols for dive planning, gas management, underwater communication and team role assignment constitute a solid platform upon which the photogrammetric study can then be constructed.

Considering the substantial time investment needed to reach such skills as a team, as well as the inherent challenges and dangers of the underwater cave environment, it seems more pragmatic to define clear and simple photogrammetric tools and Standard Operating Procedures and to teach them to experienced cave divers, than to have photogrammetry experts spend years into training to become cave divers.

\subsection{Quantitative assessment \& proof of concept}

Given the challenges having led to the design of the compact acquisition setup described in this paper, a comparative metrics study with models obtained via a traditional full-frame sensor camera would only make limited sense. If the environment can accommodate a mirrorless or DSLR system with professional quality lenses, the resulting images and photogrammetric model will undoubtedly be of superior quality - but the compact setup has mainly been built for and tested in environments where a bulkier setup would not be usable.

Table 3 summarizes key metrics for four models generated with Agisoft Metashape Pro from images acquired with the new compact setup in study areas between two and four square meters - and constitutes a first phase of practical applications considered to be a proof of concept by the authors. 


\begin{tabular}{|l|c|c|c|c|}
\hline Model & $\begin{array}{c}\text { Aligned } \\
\text { pictures }\end{array}$ & $\begin{array}{c}\text { Tie- } \\
\text { Points }\end{array}$ & $\begin{array}{c}\text { Cloud } \\
\text { points }\end{array}$ & $\begin{array}{c}\text { Mesh } \\
\text { faces }\end{array}$ \\
\hline KBASX & $221 / 221$ & 269,970 & $54,204,178$ & $2,826,914$ \\
\hline KBA007 & $761 / 761$ & 855,817 & $19,543,663$ & $1,130,163$ \\
\hline KBATAP & $445 / 445$ & 495,090 & $161,517,904$ & $8,792,001$ \\
\hline KBASL2 & $709 / 709$ & 615,236 & $126,422,601$ & $8,170,352$ \\
\hline
\end{tabular}

Table 3. Metrics for four models generated from images acquired with the new compact setup (CINDAQ)

\section{CONCLUSION}

The compact setup and standard operating procedures developed by the authors and extensively tested during fieldwork constitute a new image acquisition tool which appears well-suited for the environmental constraints of the local underwater caves: sediments, percolation, restrictions in the passageways leading to the objects and features to be documented, but also cracks, low ceilings and overhangs at the locations of the study site. Not only does the new configuration open possibilities to document sites that could not be studied using traditional setups, its simplicity and reproducibility also unlocks new potential for scaling up the data collecting process. Increased manoeuvrability can enhance the speed of the overall workflow and contribute to the safety of the dive team by reducing task overloading. Finally, it should be pointed out that the new setup does not aim to replace existing, high-resolution full-frame sensor cameras, which can still be used to document sites where they constitute a realistic option, or to collect further data on eligible sites already explored with the compact setup at the request of the scientific research team.

Following the successful proof of concept of the compact setup for the data acquisition part of the photogrammetric process, the next step for the authors' research will be to define image preprocessing SOPs matching the new configuration, including but not limited to color improvement techniques, image masking and image enhancement filters.

\section{ACKNOWLEDGEMENTS}

Research is conducted under the auspices of the Subdireccion de Arqueologia Subacuatica (SAS) del Instituto Nacional de Antropologia e Historia (INAH) and under the direction and with the cooperation of Roberto Junco, Silvina Vigliani, and Helena Barba. From Centro INAH Quintana Roo, we acknowledge director Margarito Molina and Carmen Rojas. Support for CINDAQ has been generously provided by Dr. Robert Lourie and facilitated by the Friends of Mexican Development Foundation. We wish to thank the following individuals and entities for their invaluable contribution to our efforts: Danylo Drohobytsky, Falko Kuester, Eric Lo, Vid Petrovic, Brian Strauss, Daniel Ponce-Taylor, the Ejido Jose Maria Pino Suarez, the Strauss Family Foundation, Joyce and Lester Coleman, Mayakoba Golf Classic / Tequila Patron, Rami Shakarchi, Global Underwater Explorers, Halcyon, Suex, Zero Gravity, and a special acknowledgement to the late Pilar Luna Erreguerena.

\section{REFERENCES}

Alvarez, L.W., Alvarez, W., Asaro, F., Michel, H.V., 1980: Extraterrestrial cause for the Cretaceous-Tertiary extinction: experimental results and theoretical interpretation. Science, 208(4448), 1095-1108

Bauer-Gottwein, P., Gondwe B.R.N., Charvet G, Marín, L.E., Rebolledo-Vieyra, M., Merediz-Alonso, G., 2011: The Yucatán Peninsula karst aquifer, Mexico. Hydrogeology Journal, 19: 507524, DOI 10.1007/s10040-010-0699-5
Drap, P., 2012. Underwater Photogrammetry for Archaeology. In Special Applications of Photogrammetry, pp. 111-136, InTech, Slavka, Croatia.

Drap, P., Merad, D., Boï, J.M., Mahiddine, A., Peloso, D., Chemisky, B., Seguin, E., Alcala, F., Bianchimani, O., 2014. Underwater multimodal survey: merging optical and acoustic data. In Underwater Seascapes, pp. 221-238, Springer, Cham.

Jablonski, J., 2001: Beyond the Daylight Zone - The Fundamentals of Cave Diving. Global Underwater Explorers.

MacDonald, B.L., Chatters, J.C., Reinhardt, E.G., Devos, F., Meacham, S., Rissolo, D., Rock, B., Le Maillot, C., Stalla, D., Marino, M.D., Lo, E., Luna Erreguerena, P., 2020. Paleoindian Ochre Mines in the Submerged Caves of the Yucatan Peninsula, Quintana Room, Mexico. Science Advances 6(27):1-13.

Menna, F., Nocerino, E., Fassi, F., Remondino, F., 2016. Geometric and Optic Characterization of a Hemispherical Dome Port for Underwater Photogrammetry. Sensors, 16, 48; doi: $10.3390 / \mathrm{s} 16010048$.

Menna, F., Nocerino, E., Remondino, F., 2017. Flat Versus Hemispherical Dome Ports in Underwater Photogrammetry. Int. Arch. Photogramm. Remote Sens. Spatial Inf. Sci., XLII-2-W3, 481-487. doi.org/10.5194/isprs-archives-XLII-2-W3-481-2017.

Nocerino, E., Menna, F., 2020. Photogrammetry: Linking the World across the Water Surface. J. Mar. Sci. Eng. 8, 128; doi: $10.3390 /$ jmse 8020128 .

Nocerino, E., Menna, F., Farella, E., Remondino, F., 2019. 3D Virtualization of an Underground Semi-Submerged Cave System. Int. Arch. Photogramm. Remote Sens. Spatial Inf. Sci., XLII-2-W15, 857-864. doi.org/10.5194/isprs-archives-XLII-2W15-857-2019.

Rissolo, D., Petrovic, V., Nava Blank, A., Chatters, J.C., Schubert, B., Widga, C., Bredehoeft, K., Arroyo-Cabrales, J., Luna Erreguerena, P., Kuester, F., 2018. Development of a 3D Point-based Visual Analytics Methodology to Enable Virtual Access and Taphonomic Analyses of Submerged Late Pleistocene and Early Holocene Cave Deposits of the Yucatan, Mexico. $5^{\text {th }}$ International Palaeontological Congress, Paris. Sciencesconf.org:ipc5:205505.

Schmidt, V.E., Rzhanov, Y., 2012. Measurement of Microbathymetry with a GoPro Underwater Stereo Camera Pair. 2012 Oceans, Hampton Roads, VA, 2012, pp. 1-6; doi: 10.1109/OCEANS.2012.6404786.

Smart, P.L., Beddows, P.A., Coke, J., Doerr, S., Smith, S., and Whitaker, F.F., 2006: Cave Development on the Caribbean coast of the Yucatan Peninsula, Quintana Roo, Mexico. Geological Society of America, Special Paper, 404, DOI: $10.1130 / 2006.2404(10)$

Vázquez-Domínguez, E, Arita, H., 2010: The Yucatan peninsula: biogeographical history 65 million years in the making. Ecography 33: 212-219 doi: 10.1111/j.1600-0587.2009.06293.x

Weidner, N., 2017. Underwater Cave Mapping and Reconstruction Using Stereo Vision. Master's Thesis. Scholarcommons.sc.edu/etd/4353 (15 January 2021). 